\title{
Configuraciones subjetivas de psicólogos ante la vulnerabilidad escolar: el programa HPV
}

\author{
Subjective Configurations of Psychologists Facing School Vulnerability: The HPV Program
}

\author{
José Sebastián Sandoval Díaz, Gabriela Barriga Saavedra ${ }^{\mathrm{b}}$, Catherine Wylie Beltran ${ }^{\mathrm{b}}$, \\ David Cuadra Martínez ${ }^{\mathrm{c}}$, Diego Palacios Díaz ${ }^{\mathrm{d}}, \&$ Roberto Storey Meza ${ }^{\mathrm{b}}$ \\ ${ }^{a}$ Universidad del Bío-Bío, Chillán, Chile \\ ${ }^{b}$ Universidad de Tarapacá, Arica, Chile \\ ${ }^{c}$ Universidad de Atacama, Copiapó, Chile \\ ${ }^{d}$ Universidad de Chile, Santiago, Chile
}

El presente estudio tuvo como objetivo conocer las configuraciones subjetivas sobre la vulnerabilidad escolar de psicólogos pertenecientes al programa Habilidades para la vida de la ciudad de Arica, Chile, así como también la autopercepción del rol profesional ejercido en el programa desde los propios actores interpelados. Para esto se utilizó un diseño cualitativo de corte fenomenológico. Para la selección de participantes se empleó un muestreo intencionado. En cuanto a la producción de datos, se aplicaron entrevistas en profundidad y grupos de discusión cuyo producto fue analizado con la estrategia de codificación de la teoría fundamentada. Los resultados se dividen en dos temas principales: condiciones estructurales que determinan la subjetividad del psicólogo dentro de la escuela; y, configuraciones subjetivas de los psicólogos. Se identificaron subcategorías en cada tema de análisis, las cuales son ilustradas por los datos narrativos. Asimismo, se discuten y valoran las implicancias en la incorporación de la subjetividad de y desde los propios psicólogos que se desempeñan en contextos educativos.

Palabras clave: configuración subjetiva, psicólogos educativos, vulnerabilidad escolar, programa Habilidades para la vida.

The present study aimed to understand the subjective configurations on school vulnerability of psychologists belonging to the program Habilidades para la Vida (life skills) of the city of Arica, Chile, as well as the self-perception of the professional role played in the program, from the actors themselves. In order to achieve this goal, phenomenological qualitative design was used, selecting a purposive sampling. Qualitative data collection was produced via in-depth interviews which were analyzed through strategies of codification based on grounded theory. The results are split into two main subjects: Structural conditions that determine the subjectivity of the psychologist within the school; and subjective configurations of psychologists. Five subcategories were identified inside each category of analysis and are illustrated by their narrative stories. Additionally, implications connected with the incorporation of that subjectivity experience of and from the psychologists who work in educational contexts are discussed and evaluated. Keywords: subjective configuration, educational psychologists, school vulnerability, life skills program.

Agradecimientos: Tesis de licenciatura de Psicología, Universidad de Tarapacá, Arica, Chile.

Contacto: J. S. Sandoval Díaz. Escuela de Psicología y Grupo de Investigación de Género, Ciudadanía y Equidad, Universidad del Bío-Bío, Andrés Bello 720, Chillán, Chile. Correo electrónico: jsandoval@ubiobio.cl

Cómo citar: Sandoval Díaz, J. S., Barriga Saavedra, G., Wylie Beltran, C., Cuadra Martínez, D., Palacios Díaz, D., \& Storey Meza, R. (2018). Configuraciones subjetivas de psicólogos ante la vulnerabilidad escolar: el programa HPV. Revista de Psicología, 27(1), 1-13.

http://dx.doi.org/10.5354/0719-0581.2018.50757 


\section{Introducción}

En la formación disciplinar del psicólogo/a, ciertas disposiciones profesionales se adquieren a través de la experiencia del "aprender haciendo", denominadas en la actualidad bajo el nombre de competencias (Juliá, 2006). Roe (2002) define las competencias como un conjunto de habilidades, conocimientos y actitudes aprendidas para desarrollar una determinada tarea o rol. Sin embargo, existirían discontinuidades entre las competencias esperadas entre los denominados psicólogos generalistas frente aquellos que se desempeñan en contextos educativos (Juliá, 2006).

Desde una perspectiva formativa, Avendaño (1996) ha señalado que los currículos de pregrado en psicología de Chile han tendido a presentar un énfasis en los objetivos cognitivos-instrumentales por sobre los ético-actitudinales. Desde el campo profesional, para García Costa, Carrasco Sanhueza, Mendoza Barra, y Pérez Villalobos (2012), las funciones demandadas a los psicólogos educacionales son variadas, tales como inclusión-integración, mejoramiento del clima y convivencia, y orientaciones pedagógicas-curriculares.

La psicología educacional en Chile nace para abordar, principalmente, problemas educativos (Baltar De Andrade \& Carrasco Aguilar, 2013), siendo una de las primeras áreas de desarrollo de esta disciplina (Urzúa, Vera-Villarroel, Zúñiga, \& Salas, 2015). De hecho, el primer laboratorio de psicología científica se crea en la Escuela Normal de Copiapó, a cargo del educador Rómulo J. Peña (Salas Contreras, 2012). Posterior a esto, comienza un desarrollo cada vez mayor orientada hacia el abordaje de problemas de aprendizaje en las escuelas, y paralelamente a la investigación y formación de profesionales, por ejemplo, con la creación de departamentos y asignaturas vinculadas con la psicología educacional en el año 1941 (Salas \& Inzunza, 2013). Actualmente, el área se encuentra muy ligada a las políticas educativas y la formación profesional de competencias genéricas y específicas, además de discusiones epistemológicas del conocimiento que se genera en esta área en cuestión (Baltar De Andrade \& Carrasco Aguilar, 2013).

Respecto a las demandas laborales del psicólogo escolar, Ossa (2006) ha señalado que sus funciones específicas han tendido a estar definidas por directores y sostenedores, generando una instrumentalización de su rol con base en las necesidades de las direcciones administrativas. Desde una perspectiva crítica, diversos autores (Baltar, 2003; Barraza López, 2015; García Costa et al., 2012) consideran que el sistema educativo ha configurado el desempeño del psicólogo bajo una orientación de tipo más clínica que escolar, centrándose mayormente en las dificultades adaptativas funcionales. Esta individualización del "alumno-problema", en algunos casos ha tendido a reproducir una comprensión psicologizante de los fenómenos escolares (Redondo, 2012), omitiendo tanto los procesos socioculturales e institucionales de macronivel, como la organización interaccional del aula, a micronivel (Vidal Tamayo, 2007). Por otro lado, Cornejo identifica como una de las dificultades principales, la escasa autocrítica reflexiva de los psicólogos hacia sus roles y prácticas educativas (Diversidad Educativa, 2007). A pesar de este encapsulamiento irreflexivo de tipo clínico-psicométrico (López, Carrasco, Morales, \& Ayala, 2011), su amplia incorporación en las escuelas ha posibilitado nuevas áreas de desempeño, tales como la vinculada a las áreas psicosociales de inclusión-integración, por medio de los proyectos de integración escolar (PIE) y de reducción de la vulnerabilidad, por medio del programa de Subvención Escolar (SEP) y el programa Habilidades para la vida (HPV) de la Junta Nacional de Auxilio Escolar y Becas (Junaeb).

No obstante, sin negar las dimensiones histórico-estructurales que relevan las perspectivas críticas-reproduccionistas (Giroux, 1992), estas han tendido a soslayar la propia subjetividad agentivaresistente de los psicólogos, reduciéndolos a meros agentes reproductores de prácticas clínicas-psicométricas. Así, se torna necesario realizar una lectura desde las propias prácticas efectivas narradas de psicólogo/as insertos en programas educativos, asumiendo una perspectiva dialéctica situada entre la subjetividad del individuo concreto y el contexto dinámico de las escuelas. A partir de esto, el presente estudio busca responder a dos objetivos: a) conocer las configuraciones subjetivas sobre la vulnerabilidad escolar sostenida por psicólogos/as educativos del programa HPV; $y, b)$ conocer la autopercepción del rol profesional ejercido en el programa, desde el imaginario de los propios actores interpelados.

Para responder, al menos tentativamente, los objetivos planteados, el escrito se estructura de la 
siguiente manera. En primer lugar, describiremos las condiciones de posibilidad que instalan el saber-poder de la vulnerabilidad como campo de inteligibilidad e intervención psicológico. En un segundo momento, analizaremos la noción de vulnerabilidad educativa, no solo desde su conceptualización abstracta, sino a partir de la concreción programática del modelo de factores protectores y de riesgo promovido por la Junaeb. En un tercer momento, abordaremos el papel que tiene la subjetividad, en la formación y actuación del psicólogo educativo en contextos vulnerables, relevando las categorías de configuración y sentido subjetivo.

\section{Vulnerabilidad escolar}

Nociones como pobreza y vulnerabilidad, se han tornado parte integral de los discursos y políticas educativas de las últimas décadas (Dean, 1999; Rose, 1999). Variadas políticas públicas han enfatizado el papel de conceptos tales como equidad, justicia social e igualdad de oportunidades, buscando así democratizar e introducir mecanismos correctivos a las desigualdades estructurales del continente (Grinberg, Infante, Matus, \& Vizcarra, 2014). A partir de esta problemática, han emergido una serie de programas educativos, que introducen nuevas modalidades de caracterización, regulación e intervención, en población categorizada como vulnerable (Ligueño Espinoza, Palacios Díaz, \& Sandoval Díaz, 2018). Lo vulnerable ha sido explorado y caracterizado desde múltiples perspectivas, adoptando diversas funciones y formas (Ruiz Rivera, 2012). Para Cornejo (2005), "este concepto, tradicionalmente vinculado a la condición socioeconómica, progresivamente se posiciona para dar cuenta de procesos colectivos e individuales de acumulación de daños" (p. 19). Dryfoos (1991) ha operacionalizado el constructo, identificando cinco factores vinculados a la vulnerabilidad adolescente: 1) características sociodemográficas (sexo, edad, etnicidad, educación de los padres, exposición rural o urbana, etc.);2) características individuales (nivel educativo, notas en la escuela, espiritualidad, personalidad, etc.); 3) características familiares (grado de cohesión, flexibilidad, calidad de la comunicación, etc.); 4) hacia el plano comunitario, el cual se subdivide en (a) sistema educacional; (b) relacional de pares; y (c) medios de comunicación de masas; 5) factores macroeconómicos (situación de empleo, oportunidades educacionales, nivel de ingreso, etc.). De acuerdo con esto, la concepción de vulnerabilidad educativa se encuentra inevitablemente marcada por los lineamientos de la vulnerabilidad social preexistente (Sabuda, 2010), actuando sobre el desempeño educativo y adaptación escolar (Giberti, Garaventa, \& Lamberti, 2005). En el marco del contexto educativo chileno, esta noción ha sido instalada por Junaeb, institución que focaliza sus acciones e intervenciones en poblaciones susceptibles a la deserción escolar. Para esta institución, la noción de vulnerabilidad se entiende:

Como aquella condición dinámica que resulta de la interacción de una multiplicidad de factores de riesgo y protectores, que ocurren en el ciclo vital de un sujeto y que se manifiestan en conductas o hechos de mayor o menor riesgo social, económico, psicológico, cultural, ambiental y/o biológico, produciendo una desventaja comparativa entre sujetos, familias y/o comunidades (Junta Nacional de Auxilio Escolar y Becas [Junaeb], 2005, p. 14).

Este abordaje utilizado por la Junaeb es operacionalizado mediante el índice de vulnerabilidad escolar (IVE), de tal forma que los colegios son clasificados mediante el porcentaje de estudiantes definidos como prioritarios por el Sistema Nacional de Asignación con Equidad para Becas; lo que permite distinguir niveles de vulnerabilidad escolar (Ñanculeo Raguileo, 2014). Este índice se calcula según la estimación del porcentaje ponderado de factores de riesgo, tales como: necesidad médica, déficit del peso para la edad, pobreza, entre otras (Infante, Matus, Paulsen, Salazar, \& Vizcarra, 2013). Desde el punto de vista interventivo, este organismo se encarga de la promoción y organización del Programa de Alimentación Escolar (PAE) y otros auxilios por medio de becas y programas de salud, entre los cuales está contemplado el HPV, en el cual se enfoca la presente investigación.

El Programa Habilidades para la Vida (HPV). Este programa surge en el año 1995, como un intento para establecer líneas de apoyo al desarrollo psicosocial del estudiantado, en pos de evitar la deserción escolar. El programa está dirigido a estudiantes de educación parvularia y de enseñanza básica (primer y segundo ciclo), a los apoderados y al equipo docente de establecimientos 
educacionales, sean municipales o particulares subvencionados caracterizados como vulnerables por el IVE (Junaeb, 2012). Los colegios particulares subvencionados postulan a través de la gestión de proyectos que son enviados a la Dirección Regional de Junaeb y, en caso de ser aprobados y puestos en marcha, tienen un plazo de ejecución y duración de tres años, con posibilidades de continuidad, mientras que para la dependencia municipal se implementa en todos los colegios sin postulación de por medio (Junaeb, 2012). El HPV cuenta con una clasificación de seis unidades de intervención que no tienen una consecución necesariamente lineal y que pueden trabajarse independientemente y/o simultáneamente: (1) promoción del bienestar y desarrollo psicosocial en la comunidad educativa; (2) detección de problemas psicosociales y conductas de riesgo por medio de perfiles obtenidos tras la aplicación de los instrumentos para padres (Pediatric Symptom Checklist [PSC]) $\mathrm{y}$ docentes (Teachers Observation of Classroom Adaptation [TOCA-RR]); (3) prevención de conductas de riesgo; (4) derivación y seguimiento de niños detectados con problemas de salud mental y/o psicosociales, la cual junto con la (5) implican reforzar la red local que facilite la coordinación con la escuela, centros o programas de apoyo psicosocial existentes; y (6) la unidad de evaluación y seguimiento de la red comunal de apoyo, que busca regular permanentemente la efectividad del modelo de intervención propuesto (Junaeb, 2012). La aplicación del programa ha permitido la obtención de cifras relevantes a partir del informe de gestión integral del año 2011 sobre la ejecución a nivel nacional del programa (Leiva et al., 2015). En el ciclo HPV-1 se estima el total de 491.188 participantes directos del programa: 265.706 niños, a 212.570 padres y 12.912 profesores; mientras que en el ciclo HPV-2 participaron 16.371 estudiantes, 13.098 padres y 532 profesores (Leiva et al., 2015).

A escala local, entre los profesionales que realizan las intervenciones, se menciona la presencia de psicólogos, psicopedagogos, trabajadores sociales, educadoras de párvulo e ingenieros informáticos (Dirección de Administración Municipal de Educación [Daem], s.f), quienes deben realizar planificaciones semestrales de actividades con padres, profesores, equipos de gestión de las escuelas y niños/as con perfiles de riesgo (Junaeb, 2011). De esta descripción se deriva que el psicólogo educativo parece ser una suerte de puente entre la psicología de la salud y la escuela, extrayendo de la primera su particular mirada en la promoción y prevención de la salud mental, por medio de métodos y técnicas predominantemente psicométricos, objetivando lo problemático a partir de la factorización de riesgos del entorno familiar (Baltar De Andrade \& Carrasco Aguilar, 2013).

Por lo tanto, si objetivamos la vulnerabilidad escolar en programas que han propuesto abordarla desde la factorización de riesgos, parece legítimo preguntarse por la génesis social y cultural de estos dispositivos que regulan las formas de intervenir sobre lo caracterizado como vulnerable (Ligueño Espinoza et al., 2018). Por otro lado, si la abordamos desde el saber técnico disciplinar, parece necesario reconocer la diversidad de enfoques que propone el constructo. Comprender estas formas de concebir la vulnerabilidad implica reflexionar sobre la dimensión subjetiva, que no ha sido del todo considerada, la cual incluye las subdimensiones cognitivas-afectivas que dan sentido a las prácticas disciplinarias de psicólogo/as al interior de las escuelas.

\section{La subjetividad: configuraciones y sentido sub- jetivo}

Para González Rey (2000) la subjetividad es determinada socialmente, pero no por un determinismo social externo, sino en un proceso de constitución que integra de forma simultánea lo social y lo individual. Este sistema complejo de significaciones y sentidos subjetivos producidos en la vida cultural humana de tipo procesual, plurideterminado, contradictorio, en constante desarrollo, y sensible a la cualidad de los momentos actuales, representa un papel esencial en las diferentes acciones a desplegar del sujeto concreto. Para González Rey (2002) esta subjetividad individual es producida en espacios sociales constituidos históricamente; por tanto, en la génesis de esta "se encuentran los espacios de una determinada subjetividad social que anteceden la organización del sujeto psicológico, quien aparece en su ontogenia como un momento de un escenario social constituido en el curso de su propia vida" (González Rey, 2002, pp. 180-181). El autor utiliza el término de configuración subjetiva para nominar las unidades dinámicas constitutivas de la personalidad del sujeto concreto, que define de la siguiente manera: 
Producciones de un sujeto en acción que está situado en múltiples tramas sociales simultáneas que aparecen como objetividades recreadas por su imaginación. La configuración subjetiva integra esa multiespacialidad y multitemporalidad de la persona, dimensiones que aparecen en los sentidos subjetivos que definen el aquí y ahora de la misma, definiendo un presente que nunca está preso en la condición objetiva de la presencia (Gonzáles Rey, 2013, p. 37).

La subjetividad, entonces, se configura en un individuo que actúa como sujeto gracias a su condición subjetiva y a las prácticas sociales constitutivas y constituyentes de este.

La manera en que los sujetos se constituyen, se moldean a sí mismos mediante prácticas y técnicas que ejercen sobre sí y que, no obstante, no son inventadas por ellos, sino que son esquemas de percepción y de actuación que les son propuestos o impuestos por su cultura, su sociedad, su grupo social. Técnicas y prácticas a través de las cuales los individuos se moldean activamente al ocuparse de sí y de los otros, y que los hacen aptos para actuar tanto en el plano individual como en el plano político y social. Individuos capaces por tanto de resistir, de oponerse y de modificar las tendencias existentes en nuestras sociedades actuales hacia la uniformización y la dominación (Ball, 1991, p. 13).

A partir de esto, en el trabajo que aquí se presenta se busca responder a las siguientes preguntas de investigación: 1) ¿cuáles son las configuraciones subjetivas de vulnerabilidad escolar de psicólogos educativos del programa HPV?, y 2) ¿cuál es la autopercepción que los profesionales manifiestan sobre su rol en las escuelas?

\section{Método}

La metodología cualitativa utilizada se fundamenta en una epistemología fenomenológica, la que busca comprender los sentidos y significados desde las propias perspectivas de los agentes involucrados (Flick, 2007), bajo un diseño de estudio flexible (Vasilachis de Gialdino, 2006). Con el objetivo de integrar criterios de validación cruzada y rigor sofisticado (Denzin, 1989), se incorporó tanto la triangulación intramétodo (Given, 2008) al complementar dos técnicas cualitativas de producción de datos orales (entrevistas y grupos de discusión), como el criterio de auditabilidad (Cornejo \& Salas, 2011) en el proceso de análisis de datos.

\section{Participantes y criterios de muestreo}

Programa HPV Arica. La implementación del programa HPV en Arica comenzó en el año 2002, a cargo del Daem, trabajando de forma directa con los colegios municipales caracterizados como vulnerables. En cuanto a los establecimientos particulares subvencionados, el programa es ejecutado por el Departamento de Psicología de la Universidad de Tarapacá (UTA), desde el año 2010. Al año 2016, son 32 los establecimientos beneficiarios del programa a nivel municipal (Daem, s.f.) y 17 particulares subvencionados. A nivel municipal (Daem), el equipo está conformado por seis psicólogos, quienes tienen funciones en primer y segundo ciclo básico; y en los particulares subvencionados, el equipo está conformado por cinco psicólogos para ambos ciclos. Respecto a la selección de participantes, se realizó un muestreo intencionado (Flick, 2007). En total participaron 11 psicólogos, cuatro coordinadoras de cada ciclo y la encargada regional del programa. Respecto a la trayectoria de los participantes seleccionados, la totalidad de ellos realizaron sus estudios en la carrera de psicología de la UTA, la que declara en su perfil de egreso una formación generalista, orientada a los campos profesionales clínico, educacional, organizacional-laboral, social-comunitario, y académico (Universidad de Tarapacá, 2018). A su vez, gran parte de los entrevistados menciona que este es su primer trabajo, al cual accedieron por disponibilidad laboral más que por interés personal (la mayoría declara interés en otras áreas, tales como clínica, laboral y jurídica).

\section{Técnicas de producción de información}

Se utilizaron dos tipos de técnicas habladas: entrevista en profundidad y grupos de discusión. La primera tuvo como objetivo reconstruir el sentido subjetivo narrado de los participantes. La segunda se utilizó "como un método casi naturalista para estudiar la generación de representaciones sociales o conocimiento social en general" (Flick, 2007 p. 135). Ambas técnicas se implementaron con base en un guion temático que consideró los siguientes tópicos: (a) políticas educativas y programa HPV, 
(b) funcionamiento del programa, y (c) rol del psicólogo educacional desde el programa. La opción metodológica de triangular ambos instrumentos tuvo como objetivo lograr mayor validez de criterio, además de contrastar y profundizar en las configuraciones subjetivas individuales con base en un conocimiento subjetivo social (Banchs, 2000; Catalán Ahumada, 2010).

\section{Procedimientos}

La información fue producida entre los meses de noviembre y diciembre del año 2015. Previa aplicación, se realizó la entrega de una carta formal de participación a cada entidad. Posteriormente, se les hizo entrega de un consentimiento informado a cada uno de los participantes. Se realizó un total de cinco entrevistas en profundidad y tres grupos de discusión en el siguiente orden: para el caso del HPV-Daem se realizaron dos grupos de discusión, para primer y segundo ciclo respectivamente, y dos entrevistas a las coordinadoras del programa HPV-1 y HPV-2 en las dependencias del Daem. Para el caso de HPV-UTA, se realizó un grupo de discusión con los psicólogos ejecutores y dos entrevistas a las coordinadoras del programa HPV-1 y HPV-2 en las dependencias de la UTA. Por último, se realizó una entrevista a la coordinadora regional del programa HPV de Junaeb. Toda la información fue registrada en audio, la cual posteriormente fue transcrita íntegramente. La duración promedio de las entrevistas fluctuó entre 60 a 90 minutos, mientras que los grupos de discusión presentaron una duración promedio de 90 minutos.

\section{Técnica de análisis de información}

Una vez transcrita la información, se utilizó la teoría fundamentada (Strauss \& Corbin, 2002), como estrategia de análisis de la información, apoyado en el software ATLAS.ti versión 7. La versión utilizada de la teoría fundamentada es la straussiana, "orientada a un trabajo de investigación más enraizado en la descripción interpretativa que en la construcción de teoría formal emergente" (Carrero, Soriano, \& Trinidad, 2012, p. 19). Para ello, se utilizó dos niveles de análisis: codificación abierta y codificación axial.

El análisis de codificación abierta, proceso en el cual los conceptos, dimensiones y propiedades emergen a partir de los datos, proporcionó 77 códigos clasificados en vivo y teóricos. La clasificación de los códigos principales conformó siete familias, en la cual se basó el análisis línea a línea de las transcripciones (tabla 1). Posteriormente, se realizó la codificación axial, definida como el proceso de identificación de relaciones entre las categorías obtenidas en la codificación abierta. En esta instancia se agruparon las familias de códigos en dos categorías temáticas: condiciones estructurales que determinan la subjetividad al interior de las escuelas, y configuración subjetiva de los psicólogo/as. Por último, con el objetivo de incrementar la validez y rigor interpretativo en el proceso de codificación, se incorporó el criterio de auditabilidad continua (Cornejo \& Salas, 2011), llevado a cabo en sesiones quincenales de equipo.

Tabla 1

\begin{tabular}{cl}
\hline $\mathrm{N}^{\circ}$ de Códigos & \multicolumn{1}{c}{ Familias } \\
\hline 7 & Programa Habilidades Para la Vida \\
4 & Junaeb \\
4 & Competencias del psicólogo educacional \\
4 & Tipos de vulnerabilidad \\
2 & Instrumentos Habilidades Para la Vida \\
3 & Rol del psicólogo dentro del programa Habilidades para la vida en la localidad de Arica \\
3 & Características de los colegios \\
\hline
\end{tabular}

\section{Resultados}

A continuación se presentan los resultados divididos en dos categorías principales. Además, se identificaron subcategorías para cada categoría principal, las que son ilustradas por los datos narrativos de los participantes.

Condiciones estructurales que determinan la subjetividad del psicólogo dentro de la escuela 
Este eje presenta la percepción de psicólogos hacia el sistema educacional. De este eje se desglosan dos subtemas: desarrollo de habilidades psicosociales $\mathrm{v} / \mathrm{s}$ rendimiento académico escolar, y dispositivo del programa HPV. En primer lugar, desde la perspectiva de los participantes, el HPV no se incorpora íntegramente a la escuela como otros tipos de programas, tales como PIE y SEP, sino que, más bien, opera como un programa de apoyo externo. Por otro lado, este programa psicosocial se presenta como una medida de apoyo a la salud mental escolar, trabajando con metodologías adaptadas a las características de los establecimientos educacionales. Así, el psicólogo, en lo nominativo, se posiciona como un agente protector que busca generar herramientas para mitigar la vulnerabilidad estudiantil.

Competencias psicosociales $\mathbf{v} / \mathbf{s}$ rendimiento académico. En este subeje los psicólogos perciben un conflicto respecto a los fines educativos promovidos por el sistema educacional. Explicitan que los establecimientos trabajan con base en el currículo escolar, el cual (sobre)enfatiza las dimensiones académicas, tales como el rendimiento y la excelencia académica, por sobre las dimensiones psicosociales, tales como el clima escolar, motivación y bienestar subjetivo. Esta sobrepriorización limita tanto el acceso a espacios de trabajo efectivo, como el desarrollo óptimo de las actividades programadas. Este énfasis en lo cognitivo-instrumental se acrecienta en la importancia que adquieren los resultados arrojados por pruebas de medición estandarizada, tales como el Sistema de Medición de Calidad de la Educación (Simce). Por lo tanto, para los psicólogos, esta perspectiva presenta una limitada concepción del desempeño escolar, relegando a un segundo plano las distintas dimensiones psicosociales vinculadas al bienestar y desarrollo educativo.

Hay un sesgo en el ámbito educacional que tiene que ver también con lo que yo llamo la obsesión Simce, que para mí es casi enfermiza esa obsesión de subir los puntajes Simce, pero subir los puntajes como sea, no importa a qué costo ni de qué forma, ni si perdura o no perdura en el tiempo, y etcétera, etcétera... Pero no sé si se está pensando efectivamente en que los niños mejoren el aprendizaje. Es la sensación que tengo, o quizás un sesgo que tengo para juzgar (psicóloga HPV-2).

Las escuelas están muy enfocadas en lo académico, en el rendimiento, en las notas, en el posicionamiento, en el Simce y cualquier otro tema vinculado más con algo más psicosocial no es prioridad, como no es prioridad se le entrega el menor tiempo posible, se le entrega, pero se hace porque siempre hay programas que hacen esto y que están rondando en los colegios para hacerlo, pero el tiempo y la calidad que se entrega a ese tipo de información es bajo ... Yo no sé qué piensan, pero ven el desempeño escolar como muy alejado del bienestar psicosocial, emocional, lo ven como un tema aparte (psicólogo HPV-1).

El programa HPV como dispositivo. Este subeje aborda los sentidos asignados a la vulnerabilidad, la cual es vaga y fragmentada ante los distintos tipos de riesgos. En primer lugar, comprenden la vulnerabilidad como una noción operativa con fines prácticos, es decir, para intervenir tempranamente sobre el estudiantado con perfil de riesgo, el que es producido por dos tecnologías métricas: mediante el IVE del establecimiento educacional y mediante la detección por escalas psicométricas (TOCA-RR y PSC). Esta confianza ciega en los instrumentos se ve acompañada por un desconocimiento generalizado respecto al contenido declarativo e implicancias prácticas de lo caracterizado como vulnerable. A partir de esto, se entiende que los psicólogos no reflexionan profundamente sobre sus disposiciones y prácticas, sino que, más bien, utilizan heurísticos vagos de fragilidad socioeconómica o pobreza, derivados del régimen de verdad ingenuo de los instrumentos psicométricos utilizados. Así lo expresan algunos participantes del estudio:

Porque tenemos la mirada de que, obviamente, intervenciones tempranas en el tiempo van a tener mayor efecto que intervenciones más tardías, entonces por lo mismo tratamos de trabajar, detectar problemas y perfiles de riesgos cuando están recién iniciando, de hecho, la primera aplicación de PSC que se realiza es en prekínder, porque ya necesitamos saber ya cómo vienen los niños desde su hogar, porque obviamente todavía no han tenido ningún sesgo escolar, podríamos decir que están ensuciando 
el instrumento, de hecho, en prekínder la educadora no responde el TOCA-RR, solamente lo responden los papás (psicóloga HPV-1).

Es vulnerabilidad como a todo nivel, porque es vulnerabilidad económica, vulnerabilidad a nivel cultural, social, cachay... Y nosotros, como programa, no decidimos qué escuelas se intervienen, a nosotros se nos dice qué escuelas tenemos que intervenir y se nos dice eso basado en los IVE que arroja cada escuela, si bien nosotros, bueno, a nivel municipal todas las escuelas tienen sobre un $90 \%$ del IVE, eso nos indica que son escuelas altamente vulnerables a nivel tanto económico, nivel social y todos lo que les comentaba, pero también tuvimos la inserción de escuelas a través del tema del polimetal que se nos subieron escuelas porque ya obviamente es una temática nacional el tema de los niños contaminados, que le suma otro riesgo a esta poblaciones, que también es un foco de atención eee...el tema de los polimetales (psicóloga HPV-1).

\section{La configuración subjetiva de los psicólogo/as}

Este tema presenta el rol autopercibido de los psicólogos hacia su propio quehacer en el programa. De este tema se desglosan tres subtemas: competencias relevadas para el trabajo efectivo en la escuela; autonomía relativa ante las demandas del entorno; y el mago sin magia: percepción hacia el trabajo de los psicólogos.

Competencias relevadas para el trabajo efectivo en la escuela. Los relatos evidencian que si bien se consideran importantes las competencias disciplinares formativas, aquellas que presentan una mayor valoración para un desempeño efectivo son las competencias blandas adquiridas en la cotidianeidad escolar. Entre las competencias relevadas se encuentran: innovación-creatividad, tolerancia a la frustración, iniciativa-proactividad, toma de decisiones, empatía, iniciativa, y manejo grupal.

Manejo grupal, la proactividad, el ser súper histriónica con los niños, tú no puedes llegar apagado, debes dejar tus problemas afuera porque de verdad hay mucho movimiento, de mirarlo a los ojos, de estar bien activos con ellos, porque como te digo tienen un perfil especial, ellos son súper inquietos, les cuesta tomar atención y por lo mismo nosotros le adecuamos las actividades porque por manual están las temáticas súper estructuradas (psicóloga HPV-2).

Pero claro, si bien la universidad te da como la base, pero uno más que nada es en la práctica en la que uno va aprendiendo se va formando. Yo si bien en la U tuvimos harto método y hartas matemáticas, pero a mí nadie me enseñó a hacer rendiciones financieras, ¿cachay? Entonces en la práctica, viendo, preguntando, vas aprendiendo, lo mismo la interacción que tienes con los directores, cómo trabajar con los directores, con los equipos directivos no tienes que llegar de la misma forma que llegas con los docentes, con los apoderados es lo mismo, las diferentes estrategias que tienes que buscar para poder comprometer a la escuela en el trabajo con habilidades, eso muchas veces lo vas aprendiendo en la práctica (psicóloga HPV-1).

Autonomía relativa ante las demandas del entorno. Los psicólogos sostienen la ausencia de espacios de autonomía profesional, tanto en el diseño de estrategias como en los aspectos administrativos de ejecución. Esto se expresa en situaciones específicas tales como la aplicación estándar del manual de actividades confeccionado a nivel nacional. En este punto los psicólogos concuerdan en que los objetivos son pertinentes para abordar el trabajo estudiantil, sin embargo, muchas veces las temáticas y actividades no son acordes con las características particulares de cada perfil de riesgo estudiantil ni con el contexto local de las escuelas. Esta situación ha llevado a tomar determinaciones respecto a los lineamientos centralizados del programa por ambos equipos. Una de las decisiones ha sido implementar actividades adicionales en los talleres preventivos, los cuales son aplicados con la visación previa de la coordinadora regional de Junaeb, proporcionando una autonomía relativa.

Es muy poca la autonomía que nosotros podemos tener, porque el modelo de las habilidades es súper riguroso, tanto en el tema de las unidades que componen el programa y las actividades que componen cada una de esas unidades y que nos dice claramente qué cantidad de talleres tenemos que hacer para cada unidad, de 
cuánto tiempo, de estas temáticas, con qué personas, en qué momento, entonces, en ese sentido, es súper estructurado. Lo que sí, nosotros ya quizás con la experiencia que ya llevamos en el programa, quizás como ya conocemos en la gran mayoría de las escuelas nos ha permitido ir un poco según las necesidades del establecimiento ir cambiando algunas cosas, pero más que nada es en la conversación de nosotros con la dirección regional de la Junaeb (psicóloga HPV-1).

El mago sin magia: percepción hacia el trabajo de los psicólogos. La comunidad educativa percibe al psicólogo como el agente encargado del cambio individual, con énfasis en la dimensión adaptativa conductual. Esta función casi mágica atribuida, sin embargo, es significada dentro un plano remedial inmediatista, más que en la promoción o prevención temprana que busca el programa. Sumado a esto, se percibe un desconocimiento generalizado respecto de las funciones diferenciadas que ejecutan los psicólogos del programa HPV, en relación con aquellos psicólogos con quienes se comparte espacio educativo al interior de los establecimientos, tales como PIE y SEP.

Los que no son psicólogos, los docentes, directivos, etc., tienen esa percepción de que uno como psicólogo va a cambiar al niño de inmediato, con una varita mágica, entonces lo que hacemos nosotros son talleres preventivos, o sea, evitar que luego desarrollan estas conductas problemáticas y potenciando los factores protectores (psicólogo HPV-1).

Algunas veces los profesores nos piden casi que entre el niño desordenado y salga todo respetuoso y ordenado de un momento a otro. Yo creo que, la verdad, en algunos establecimientos no tienen claro nuestra función como programa HPV, nos ven como psicólogos no más (psicólogo HPV-2).

\section{Discusión}

De acuerdo con lo presentado, los sentidos subjetivos y prácticas de los psicólogos/as emergen de la relación dialéctica entre macroprocesos educativos y configuraciones subjetivas situadas; estas últimas constituyen el espacio personal para construir disposiciones de acción reproductoras o resistentes ante los cánones hegemónicos-normativos del sistema educacional vigente (González Rey, 2013). A su vez, los resultados evidencian cómo estas configuraciones subjetivas se van condicionando según los distintos niveles de determinación: sistema educacional nacional-comunal, programa HPV nacional, y escuelas. Estas constricciones multiespaciales y multitemporales producen oportunidades o desventajas educativas, configurando así prácticas, sentidos y una percepción normativa sobre el rol de los psicólogos.

A nivel de sistema, las demandas estructurales por mejorar los estándares de calidad, bajo el discurso neoliberal de la gestión empresarial de las escuelas (Ligueño Espinoza et al., 2018), han tendido a limitar aún más el ingreso e importancia de lo psicosocial como dimensión relevante en lo educativo. De acuerdo con esto, el espacio otorgado a la vulnerabilidad escolar evidencia una apertura a lo psicosocial en las escuelas; sin embargo, la gestión de la calidad escolar ha tendido a restar importancia a su promoción al considerarla indirectamente provechosa para la mejora de los estándares de desempeño cognitivos-instrumentales dominantes. Esta mirada restrictiva sobre la calidad educativa es puntualizada en los relatos a través de la obsesión por el Simce, la que, según los fines promovidos por el sistema, soslaya la potenciación y desarrollo de otro tipos de competencias tales como: tener y desarrollar un proyecto de vida propio; participar e influir en la sociedad; experimentar placer y emociones; y tener vínculos significativos con los demás (Castillo \& Contreras, 2014; Sandoval Díaz, Ligueño Espinoza, \& Palacios Díaz, 2015). No obstante, si bien en los últimos años se han incorporado elementos que han buscado ampliar esta concepción restrictiva de la calidad, por medio de los denominados indicadores de desarrollo personal y social, estos siguen funcionando solo como medios complementarios a los resultados de la prueba Simce (Agencia de Calidad, 2018), aspirando al mecanismo de información perfecta para los demandantes al que tiende el sistema educativo chileno.

A nivel de programa, la alta estructuración y homogenización de las metodologías del HPV ha dejado poco espacio a la flexibilidad y pertinencia local, buscando modelar un psicólogo de la salud aplicado al contexto educativo, con el fin de alcanzar el cumplimiento de los propósitos establecidos 
por Junaeb. Esta incompatibilidad o desajuste percibido en los procedimientos presenta problemas prácticos a la hora de modificar o adecuar la pertinencia de dichas estrategias, limitando aún más la capacidad de autonomía relativa de los profesionales. Lo anterior invita a discutir acerca de la formación profesional del psicólogo que ejercerá labores en las escuelas con base en programas altamente estructurados como el HPV o los PIE, en el sentido que se presenta una incongruencia entre la tan anhelada reflexividad profesional y las restricciones del ejercicio profesional.

Respecto a la vulnerabilidad escolar, los entrevistados manifiestan un conocimiento declarativo parcial y fragmentado sobre esta, guiando su accionar por el sentido práctico arrojado por las tecnologías psicométricas de detección de población en riesgo (TOCA-RR y PSC). Sin embargo, esta falta de conocimiento y de reflexividad sobre sus prácticas (Diversidad Educativa, 2007) no quita que se establezcan explicaciones causales sobre el origen e intervención sobre lo caracterizado como vulnerable, que es significado bajo dos sentidos subjetivos: desde la dimensión individual psicopatológica, y desde la perspectiva sistémica familiar, omitiendo, en gran medida, las dimensiones estructurales-procesuales que la originan (Ligueño Espinoza et al., 2018).

A su vez, predomina un sentido más ético-individual que político en el para qué de la intervención. Esto presenta concordancia con la noción de vulnerabilidad promovida por Junaeb, la que se enmarca en un discurso paliativo de la desigualdad contextual del entorno inmediato, remitiéndose estrictamente a la carencia y susceptibilidad de riesgos individuales y/o familiares (Infante et al., 2013). Este entramado discursivo sobre la vulnerabilidad ha tendido a caer en la psicologización e individualización de lo vulnerable, omitiendo sus condiciones sociohistóricas que permiten entender, por una parte, que existirían factores de orden estructural en su origen y no únicamente en los sujetos, y que la asistencialidad por parte de las instituciones del Estado, solo se limita a satisfacer necesidades básicas, no incidiendo en los factores que causan la vulnerabilidad (Infante et al., 2013).

Por lo tanto, este tipo de discurso psicologizante invisibiliza las relaciones de poder y su distribución desigual en la sociedad, haciendo emerger solo al individuo susceptible a riesgos. De acuerdo con Bourdieu (2004), consideramos relevante el desarrollo formativo de una reflexividad continua ante el sentido práctico profesional, fomentando la adquisición de las competencias de vigilancia epistemológica y problematización ante la naturalización de lo psicosocial, identificando los límites del conocimiento disciplinar, pero también sus potencialidades ante situaciones concretas. Operativamente, esto implica que tanto psicólogos/as como estudiantes de psicología logren una integración reflexiva y consciente de los saberes subjetivos, profesionales y académico-científicos del objeto de intervención (Cuadra-Martínez, Castro, \& Juliá, 2018).

Por otro lado, la percepción de la comunidad educativa hacia el rol de los psicólogos presentó dos matices: un desconocimiento respecto a las funciones programáticas que este realiza (y hacia el programa en general); y se le atribuían funciones de carácter clínico-remedial (García Costa et al., 2012). Respecto a la identidad autopercibida de los psicólogos, al parecer no existe una relación lineal entre el contexto de desempeño y la identidad profesional percibida. En esta misma línea, los profesionales resaltan la importancia de las competencias blandas (empatía, tolerancia a la frustración, manejo grupal) por sobre las competencias técnicas duras, es decir, sobre aquellas adquiridas en la formación disciplinar universitaria. Esta distancia entre lo teórico práctico o lo científico y profesional, podría tener origen en las características de los procesos formativos de los profesionales, que el enfoque por competencias de la educación superior no ha logrado resolver (Cuadra-Martínez et al., 2018). Sería relevante, además, comprender cómo estas disposiciones operan como estrategias y tácticas para abordar lo problemático de los procesos cotidianos de trabajo en el contexto educativo.

Atendiendo a ello, para investigaciones posteriores a nivel nacional, resultaría relevante comparar las cercanías / distancias entre configuraciones subjetivas de profesionales que operan sobre los otros sentidos subjetivos de la vulnerabilidad, tales como los estudiantes prioritarios de la SEP o aquellos que presentan necesidades educativas especiales del PIE, con las cuales comparten espacio al interior de las escuelas. Para Latinoamérica, creemos relevante identificar y distinguir la diferenciación de la vulnerabilidad con otras nociones colindantes, tales como pobreza y marginación. A su vez consideramos importante relevar las 
distintas expresiones e intervenciones socioculturales sobre lo vulnerable bajo el actual marco neoliberal globalizado de políticas educativas gerencialistas.

Por último, si bien no existe una propuesta unívoca en el cómo deben ser estructuradas y enmarcadas las funciones del psicólogo educativo, tanto en su formación como en lo laboral (Ossa, 2006), esto nos abre un espacio de autonomía relativa importante para que los propios psicólogo/as puedan configurar, activa y reflexivamente, sus propias razones prácticas, instituyendo sentidos subjetivos capaces de modificar sus competencias disciplinarias ante las necesidades "sentidas y dinámicas" que los propios entornos educativos demandan. Mirar hacia allá implica que el psicólogo educativo logre desarrollar una teoría compleja que guíe su acción profesional sustentada en el saber subjetivo, el saber profesional y el saber científico técnico (Cuadra-Martínez et al., 2018; Sandoval \& Cuadra, en prensa), para resolver profesionalmente problemas sociales complejos, como lo es la vulnerabilidad en contexto educativo.

\section{Referencias}

Agencia de Calidad. (2018). Indicadores de desarrollo personal y social de los estudiantes. Santiago, Chile: Agencia de Calidad.

Recuperado de https://goo.gl/ndrnQ1

Avendaño, C. (1996). Formación profesional del psicólogo. Antecedentes acerca de la formación del psicólogo en Chile. Terapia Psicológica, 26, VI(2), 43-47.

Ball. S. J. (1991). Foucault y la educación. Disciplinas y saber. Madrid, España: Morata.

Baltar, M. J. (2003). El sentido del diagnóstico psicológico escolar. Un análisis crítico y una propuesta en construcción. Psicoperspectivas, 2(1), 7-34.

Recuperado de https://goo.gl/eotdwy

Baltar De Andrade, M. J. \& Carrasco Aguilar, C. L. (2013). Re-pensando la psicología educacional en Chile: análisis crítico de su quehacer y sugerencias proyectadas. Psicología para América Latina, (24), 173-190.

Recuperado de https://goo.gl/oYF5CN

Banchs, M. A. (2000). Aproximaciones procesuales y estructurales al estudio de las representaciones sociales. Papers on Social Representations, 9, 3.13.15 .

Recuperado de https://goo.gl/7Tb2De

Barraza López, R. (2015). Perspectivas acerca del rol del psicólogo educacional: propuesta orientadora de su actuación en el ámbito escolar. Revista
Electrónica Actualidades Investigativas en Educación, 15(3), 1-21.

Recuperado de https://goo.gl/XVio3B

Bourdieu, P. (2004). Cosas dichas. Barcelona, España: Gedisa.

Carrero, V., Soriano, R. M., \& Trinidad, A. (2012). Teoría fundamentada grounded theory. El desarrollo de teoría desde la generalización conceptual. Madrid, España: Centro de Investigaciones Sociológicas.

Castillo, J. \& Contreras, D. (2014). El papel de la educación en la formación del bienestar subjetivo para el desarrollo humano. Una revisión al caso chileno. Santiago, Chile: PNUD y Unicef.

Recuperado de https://goo.gl/vxHWg7

Catalán Ahumada, J. (2010). Teorías subjetivas: aspectos teóricos y prácticos. La Serena, Chile: Editorial Universidad de La Serena.

Cornejo, R. (2005). El experimento educativo chileno 20 años después: ¿mejora la calidad de la educación con el mercado y la competencia? Revista Pluma y Pincel, (186), 19-25.

Cornejo, M. \& Salas, N. (2011). Rigor y calidad metodológicos: un reto a la investigación social cualitativa. Psicoperspectivas, 10(2), 12-34. http://doi.org/cs5s

Cuadra-Martínez, D. J., Castro, P. J., \& Juliá, M. T. (2018). Tres saberes en la formación profesional por competencias: integración de teorías subjetivas, profesionales y científicas. Formación Universitaria, 11(5).

Dean, M. (1999). Governmentality: Power and rule in modern society. London, United Kingdom: Sage.

Denzin, N. K. (1989). The research act: A theoretical introduction to sociological methods. Englewood Cliffs, New Jersey: Prentice Hall.

Dirección de Administración Municipal de Educación. (s.f.). Programa Habilidades para la Vida. Arica, Chile: Daem.

Diversidad Educativa. (2007). Los psicólogos y el futuro de nuestra educación: Entrevista a Rodrigo Cornejo. Diversidad Educativa.

Dryfoos, J. G. (1991). Adolescents at risk: Prevalence and prevention. Oxford, United Kingdom: Oxford University Press.

Flick, U. (2007). Introducción a la investigación cualitativa. Madrid, España: Morata.

García Costa, C., Carrasco Sanhueza, G., Mendoza Barra, M., \& Pérez Villalobos, C. (2012). Rol del psicólogo en establecimientos particulares pagados del Gran Concepción, Chile: un proceso de co-construcción. Estudios Pedagógicos, 38(2), 169-185. http://doi.org/cs5v

Giberti, E., Garaventa, J., \& Lamberti, S. (2005). Vulnerabilidad, desvalimiento y maltrato infantil en las organizaciones familiares. Buenos Aires, Argentina: Centro de Publicaciones Educativas y Material 
Didáctico.

Giroux, H. A. (1992). Teoría y resistencia en educación: una pedagogía para la oposición. México, México: Siglo XXI.

Given, L. M. (Ed.). (2008). The Sage encyclopedia of qualitative research methods. Thousand Oaks, California: SAGE.

González Rey, F. L. (2000) Investigación cualitativa en psicología. Rumbos y desafios. México, México: International Thomson Editores.

González Rey, F. (2002). Sujeto y subjetividad. Una aproximación histórica-cultural. México, México: Thomson.

González Rey, F. L. (2013). La subjetividad en una perspectiva cultural-histórica: avanzando sobre un legado inconcluso. Revista CS, (11), 19-42. http://dx.doi.org/10.18046/recs.i11.1565

Grinberg, S., Infante, M., Matus, C., \& Vizcarra, R. (2014). Espacios y subjetividades: narrando las trayectorias de la vulnerabilidad. Estudios Pedagógicos, 40(2), 203-219. http://doi.org/cs5w

Infante, M., Matus, C., Paulsen, A., Salazar, A., \& Vizcarra, R. (2013). Narrando la vulnerabilidad escolar: performatividad, espacio y territorio. Literatura $y$ Lingüística, (27), 281-308. http://doi.org/cs5x

Juliá, M. T. (2006). Competencias profesionales del psicólogo educacional: una tarea asociativa. Revista de Psicología, 15(2), 115-130. http://dx.doi.org/10.5354/0719-0581.2006.18417

Junta Nacional de Auxilio Escolar y Becas. (2005). Sinae Sistema Nacional de Asignación con Equidad para Becas Junaeb. Una nueva visión en la construcción de igualdad de oportunidades en la infancia. Santiago, Chile: Junaeb.

Recuperado de https://goo.gl/UkRj22

Leiva, L., George, M., Antivilo, A., Squicciarini, A. M., Simonsohn, A., Vargas, B., \& Guzmán, J. (2015). Salud mental escolar: logros de una intervención preventiva en salud mental en niños y niñas del primer ciclo de enseñanza básica. Psicoperspectivas, 14(1), 31-41.

Recuperado de https://goo.gl/CcXj2b

Ligueño Espinoza, S., Palacios Díaz, D., \& Sandoval Díaz, J. (2018). Discursos de la vulnerabilidad bajo el gerenciamiento educativo: análisis Ley de subvención escolar preferencial chilena. Revista de Investigación Psicológica, (19), 207-222.

Recuperado de https://goo.gl/ssrmqP

López, V., Carrasco, C., Morales, M., \& Ayala, A. (2011). El encapsulamiento de los psicólogos escolares y profesionales de apoyo psicosocial en la escuela. Revista Internacional Magisterio, (53), 5457.

Recuperado de https://goo.gl/R7guPY
Nanculeo Raguileo, M. A. (2014). Construcción de un índice de vulnerabilidad social para estudiantes preescolares de establecimientos subvencionados de Chile (Tesis de magíster). Universidad de Concepción, Concepción, Chile.

Recuperada de https://goo.gl/Cct2JJ

Ossa, C. (2006). Factores que estructuran el rol del psicólogo en educación especial. Revista de Psicología, 15(2) ,131-145. http://dx.doi.org/10.5354/0719-0581.2006.18418

Redondo, J. (2012). Sociedad y equidad: aportes desde la psicología. En C. Zúñiga Rivas (Ed.), Psicología, sociedad y equidad: aportes y desafios (pp. 13-22). Santiago, Chile: Colección Praxis Psicológica.

Roe, R. (2002). What makes a competent psychologist? European Psychologist, 7(3), 192-202. http://doi.org/fh9hhp

Rose, N. (1999). Powers of freedom. Reframing political thpught. Cambridge, United Kingdom: Cambridge University Press.

Ruiz Rivera, N. (2012). La definición y medición de la vulnerabilidad social. Un enfoque normativo. Investigaciones Geográficas, (77), 63-74. http://dx.doi.org/10.14350/rig.31016

Sabuda, F. G. (2010). ¿Quién es vulnerable en la escuela? Análisis territorial de rendimientos educativos y contexto sociocultural en el Partido de General Pueyrredón, Argentina. Cuadernos de Geografia: Revista Colombiana de Geografía, (18), 45-57. Recuperado de https://goo.gl/Z3oWPz

Salas, G. \& Inzunza, J. (2013). Antecedentes históricos de la psicología educacional en Chile. En C. Cornejo, P. Morales, E. Saavedra, \& G. Salas (Eds.), Aproximaciones en psicología educacional. Diversidades ante la contingencia actual (pp. 27-41). Talca, Chile: Universidad Católica del Maule.

Salas Contreras, G. (2012). La influencia europea en los inicios de la historia de la psicología en Chile. Interamerican Journal of Psychology, 46(1), 99-109. Recuperado de https://goo.gl/pMMSUr

Sandoval, J. \& Cuadra. D. (en prensa). El abordaje psicológico de la vulnerabilidad escolar desde una dimensión subjetiva: el psicólogo escolar entre la estructura y agencia. En F. Leal (Ed.), Psicólogos y psicólogas ¿Extranjeros en las escuelas? Temas de psicología educacional. Contribuciones para la formación de especialidad. (Vol. 2). Iquique, Chile: Noveduc.

Sandoval Díaz, J., Ligueño Espinoza, S., \& Palacios Díaz, D. (2015). ¿Merece el bienestar subjetivo un espacio en la escuela chilena? Revisión del concepto y propuestas de análisis para el escenario educativo actual. En E. Saavedra, G. Salas, C. Cornejo, \& P. Morales. Resiliencia y calidad de vida: la psicología educacional en diálogos con otras disciplinas (pp. 75-93). Talca, Chile: Universidad Católica del 
Maule.

Strauss, A. \& Corbin, J. (2002). Bases de la investigación cualitativa: técnicas y procedimientos para desarrollar la teoría fundamentada. Medellín, Colombia: Universidad de Antioquia.

Universidad de Tarapacá. (2018). Proceso de admisión carrera de psicología. Arica, Chile. Recuperado de https://goo.gl/DMHbCz

Urzúa, A., Vera-Villarroel, P., Zúñiga, C., \& Salas, G. (2015). Psicología en Chile: análisis de su historia, presente y futuro. Universitas Psychologica, 14(3), 1141-1158.

http://doi.org/cs62

Vasilachis de Gialdino, I. (Coord.), (2006). Estrategias de investigación cualitativa. Barcelona, España: Gedisa.

Vidal Tamayo, J. P. (2007). Aportes del psicólogo educacional a los establecimientos educativos. Trabajo presentado en el Diplomado Psicología Educacional, Universidad de Chile, Santiago, Chile.

Recuperado de https://goo.gl/qfJzk3

Fecha de recepción: 27 de enero de 2017

Fecha de aceptación: 2 de agosto de 2018 